\title{
Further delineation of Loeys-Dietz syndrome type 4 in a family with mild vascular involvement and a TGFB2 splicing mutation
}

\author{
Marco Ritelli ${ }^{1}$, Nicola Chiarelli ${ }^{1}$, Chiara Dordoni ${ }^{1}$, Stefano Quinzani ${ }^{1}$, Marina Venturini ${ }^{2}$, Roberto Maroldi ${ }^{3}$, \\ Piergiacomo Calzavara-Pinton ${ }^{2}$ and Marina Colombi ${ }^{* *}$
}

\begin{abstract}
Background: The Loeys-Dietz syndrome (LDS) is a rare autosomal dominant disorder characterized by thoracic aortic aneurysm and dissection and widespread systemic connective tissue involvement. LDS type 1 to 4 are caused by mutations in genes of the TGF- $\beta$ signaling pathway: TGFBR1 and TGFBR2 encoding the TGF- $\beta$ receptor (LDS1 and LDS2), SMAD3 encoding the TGF- $\beta$ receptor cytoplasmic effector (LDS3), and TGFB2 encoding the TGF- $\beta 2$ ligand (LDS4). LDS4 represents the mildest end of the LDS spectrum, since aneurysms are usually observed in fourth decade and the progression of the disease is slower than in the other forms.

Case presentation: We report the clinical and molecular findings of an LDS4 Italian family. Genetic testing included TGFBR1, TGFBR2, SMAD3, and TGFB2 analysis by Sanger sequencing. In order to verify the effect of the identified splice mutation, RT-PCR analysis was performed.

The proband, a 57-year-old woman, showed high palate, hypoplasic uvula, easy bruising, joint hypermobility, chronic pain, scoliosis, multiple relapsing hernias, dural ectasia, and mitral valve prolapse. Magnetic resonance angiography revealed tortuosity and ectasia of carotid, vertebral, cerebral, and segmental pulmonary arteries. Arterial aneurysm and dissection never occurred. Her 39- and 34-year-old daughters presented with a variable degree of musculoskeletal involvement. Molecular analysis disclosed the novel c.839-1G>A splice site mutation in the TGFB2 gene. This mutation activates a cryptic splice acceptor site in exon 6 leading to frameshift, premature termination codon and haploinsufficiency (p.Gly280Aspfs*41).
\end{abstract}

Conclusions: Our data confirm that loss-of-function mutations in TGFB2 gene do not always lead to aggressive vascular phenotypes and that articular and skeletal signs are prevalent, therefore suggesting that LDS4 must be considered in patients with sparse signs of LDS and related disorders also in the absence of vascular events.

Keywords: Loeys-Dietz syndrome type 4, TGFB2, TGF-ß2, Splicing mutation

\section{Background}

Thoracic Aortic Aneurysms and Dissections (TAADs) are a common cause of sudden death of young adults. The pathophysiology of TAAD is complex and multi-factorial. To date, though classic cardiovascular risk factors play a pivotal role in a majority of patients, several genes have been identified in both syndromic and nonsyndromic forms of TAAD, including FBN1 (Marfan syndrome, MFS)

\footnotetext{
* Correspondence: marina.colombi@unibs.it

'Division of Biology and Genetics, Department of Molecular and Translational Medicine, Medical Faculty, University of Brescia, Viale Europa 11, Brescia 25123, Italy

Full list of author information is available at the end of the article
}

[1], TGFBR1, TGFBR2 [2,3] and SMAD3 (Loeys-Dietz syndrome type 1 to 3, LDS) [4], SLC2A10 (Arterial tortuosity syndrome, ATS) [5], ACTA2 (TAAD with livedo reticularis and iris flocculi) [6], MYH11 (TAAD with patent ductus arteriosus) [7], and MYLK (TAAD7) [8]. Although clinical features show significant overlap, these entities differ in the extent of vascular involvement and clinical course and, as a consequence, molecular characterization has become crucial in the evaluation, counseling and management of these patients. Among the syndromic forms of TAAD, LDS is a rare autosomal dominant disorder with widespread systemic involvement and connective tissue 
disorders (CTD) signs. LDS type 1 (LDS1, MIM\#609192) and type 2 (LDS2, MIM\#610168) are caused by heterozygous mutations in the genes encoding the TGF- $\beta$ receptors type 1 and 2 , which bind TGF- $\beta$ and initiate cellular signaling $[2,3]$. The majority of individuals with LDS1 and 2 shows vascular findings with life-threatening complications, such as aortic dissection and widespread aortic aneurysms, and life expectancy is reported to be 37 years $[9,10]$. Craniofacial signs as ocular hypertelorism, bifid uvula/cleft palate or craniosynostosis and cutaneous manifestations, as velvety and translucent skin, easy bruising, atrophic scars, are also present at variable degree $[2,3,9,11]$. In the first reports, LDS patients were stratified into 2 types, depending on severity of craniofacial features (type 1) or cutaneous features (type 2). However, these findings are now believed to be part of a continuum within the LDS spectrum of disease [12]. Mutations in the SMAD3 gene, which encodes a protein critical for cellular signaling downstream of the TGF- $\beta$ receptors after ligand binding, are responsible for LDS type 3 (LDS3, MIM\#613795), also known as Aneurysms-Osteoarthritis Syndrome. LDS3 patients share phenotypic manifestations with LDS1 and 2, i.e., aortic root aneurysm, vascular dissection, skeletal deformities, and typically show osteoarthritis at young age $[13,14]$, although families without osteoarthritis are also reported [15].

Lindsay et al. and Boileau et al. identified an additional gene responsible for familial TAAD, the TGFB2 gene, which encodes the ligand TGF- $\beta 2[16,17]$. In particular, Boileau et al. discovered heterozygous mutations in 2 families and 2 sporadic cases with TAA [17], and Lindsay et al. identified TGFB2 mutations in 8 families [16]. Both groups examined aortic tissue from probands and found fragmentation of elastin fibers and deposition of collagen and proteoglycans, similar to what is observed in tissues from MFS and LDS patients. Furthermore, altered TGF- $\beta$ signaling, as judged by an increase in TGF- $\beta 1$ or TGF- $\beta 2$ protein expression and greater nuclear accumulation of phosphorylated SMAD2, was demonstrated. In TGFB2 heterozygous knockout $\left(T g f b 2^{+/-}\right)$mice Lindsay et al. found aortic root dilatation and increased TGF- $\beta$ signaling, consistent with the human disease presentation [16]. Careful clinical phenotyping of TGFB2 mutation-bearing individuals identified a variety of vascular, musculoskeletal, and other features shared by patients with either MSF or LDS, therefore, the disorder was classified as LDS4 (MIM\#614816). Features shared with MFS and LDS include aortic aneurysm, mainly in thoracic tract, pectus deformity, arachnodactyly, scoliosis, joint hypermobility, and striae distensae in young age. Features shared with LDS, but not MFS, include hypertelorism, bifid uvula, bicuspid aortic valve, mitral valve prolapse (MVP), arterial tortuosity, club feet, and thin skin with easy bruising [16].
In LDS4 aortic aneurysm presents at later onset; relatively mild aortic dilatation, mainly at the level of the sinus of Valsalva, and a lower incidence of dissection compared to the other LDS types was observed, and the reported mean age of aortic disease was 35 years [17]. Renard et al. described other 6 sporadic LDS4 patients with TGFB2 mutations, pointing out a high rate of mitral valve disease, suggesting that this might be a signature feature of the disorder that may direct molecular analysis [18]. Leutermann et al., very recently described another LDS4 family with three affected members [19]. Recently a revised LDS nosology was proposed, in particular the authors suggested that a mutation in any of these 4 genes in combination with documented aneurysms or dissection should be sufficient for the diagnosis of LDS [20].

Here, we report on a new LDS4 family with a 57year-old proband without thoracic aortic aneurysms and other major vascular complications, caused by a novel mutation in the TGFB2 gene and compare the clinical features of the affected members with those reported in the literature. Our data suggest that LDS4 must be considered in patients with sparse signs of LDS and related disorders, also in the absence of vascular events.

\section{Case presentation}

\section{Patients, materials and methods}

We describe an Italian LDS4 family with three affected members: the proposita and her 34-year-old daughter, underwent careful clinical evaluation and provided written informed consent for genetic testing. The clinical features of the 39-year-old daughter were reported by the proposita, since this daughter did not consent clinical evaluation and genetic testing, likewise both proband's parents, in the octave decade and with a referred unremarkable clinical history, could not be evaluated.

The proposita underwent extensive cardiovascular studies, including electrocardiogram (ECG), echocardiography, and brain, thoracic and abdominal magnetic resonance angiography (MRA). The 34-year-old daughter did not consent MRA study and only ECG and echocardiography with aortic root evaluation were performed. Genomic DNA was purified from blood samples using Wizard Genomic DNA purification Kit (Promega) by standard procedures. Total RNA was isolated from proband's whole blood using NucleoSpin RNA Blood Midi Kit (Macherey-Nagel) and retrotranscribed in the presence of random hexanucleotide primers and MMLV-RTase (Life Technologies) by standard procedures. Primers were designed for all coding exons, including their intron-exon boundaries, and for the $5^{\prime}$ and $3^{\prime}$ UTRs near the coding sequence (a minimum of $200 \mathrm{bp}$ ), using the Primer3 tool (http:// frodo.wi.mit.edu/). Primer sequences were checked for the absence of known variants, such as single nucleotide polymorphisms, based on dbSNP version 139 
(www.ncbi.nlm.nih.gov/projects/SNP/). In particular, all exons and intron flanking regions of TGFBR1, TGFBR2, SMAD3 and TGFB2 genes were amplified by standardized PCR using optimized genomic primer-sets and the GoTaq Ready Mix 2X (Promega) (primer sequences and amplification profiles are available upon request). After enzymatic cleanup of the PCR products by ExoSap-IT (Affymetrix), all fragments were sequenced in both orientations using the BigDye ${ }^{\circ}$ Terminator Cycle Sequencing kit protocol (Life Technologies) and the Performa DTR Ultra 96-Well Plates (EdgeBio) for PCR cleanup followed by capillary electrophoresis on the ABI3130XL Genetic analyzer (Life Technologies). The identified TGFB2 mutation was not found in 100 control individuals or in the 1000 Genomes Project or NHLBI Exome Variant Server (ESP6500) databases. Nucleotide and protein accession numbers correspond to the TGFB2 (NM_000093.3, NP_000084.3) reference sequences. The mutation was annotated according to HGVS nomenclature (www.hgvs. org/mutnomen), and the sequence description verified using Mutalyzer (www.LOVD.nl/mutalyzer). Nucleotide numbering was based on cDNA sequence numbering with +1 corresponding to the A of the ATG translation initiation codon 1 in the reference sequence. For protein numbering, +1 corresponds to the first translated amino acid. The sequences were analyzed using the Sequencher 4.9 software (www.genecodes.com). To evaluate the identified splice site mutation, we used four prediction programs (SpliceSite-Finder-like, MaxEntScan, NNSPLICE and Human Splicing Finder) in the Alamut Software version 2.4 (www.interactive-biosoftware.com). Further, to verify the effect of the splice mutation, RT-PCR was carried out by standard procedures; in particular, amplification of cDNA covering exons 5-7 of the TGFB2 gene was performed.

\section{Clinical findings}

The proposita (Figure 1A) was a 57-year-old woman with family history remarkable for CTD in her two 39and 34-year-old daughters. She was born from unrelated Italian parents after an uneventful pregnancy and delivery. Her perinatal and psychomotor development was normal. Two pregnancies resulted in the birth of two daughters. In the first pregnancy she reported premature membrane rupture at 34 weeks, and in the second a threatened abortion. The spontaneous menopause was at 50 years. Clinical history presented widespread signs of CTD. Since the childhood sub/luxations of the shoulders, wrists, knees and ankles were occurring. She suffered from acute articular rheumatism, diagnosed at 9 years, and right relapsing inguinal hernia, surgically treated at 9, 25, and 40 years. Since her twenties, she referred chronic generalized articular pain, mainly affecting her back, treated with NSAIDs. Magnetic resonance imaging (MRI), performed at 42 years, revealed dural ectasia, lumbar discal hernias (L5-S1) and hypoplasia of the twelfth ribs (Figure 1B). Clinical history also included crural hernia, surgically treated at 25 and 40 years, hiatal hernia with gastroesophageal reflux, chronic headache, gingival fragility, and easy bruising. Ectopia lentis was excluded by ophthalmologic evaluation. ECG and echocardiography, performed at 49 years for tachycardia, discovered paroxysmal supraventricular tachycardia and MVP with minimal regurgitation and normal systolic function (EF 65\%). Following this analysis she underwent cryoablation therapy. At this age the aortic root diameter was normal. On examination at 56 years, she presented with normal stature $(1,63 \mathrm{~m})$, light blue sclerae, high arched palate, micrognathia, elongated philtrum, hypoplasic uvula, doughy and hyperextensible skin over the neck, the forearm, and the elbows, old aging aspect, striae distensae over the hips, joint hypermobility according to Beighton score (9/9), and scoliosis (Figure 1A). In a clinical suspicion of LDS, a brain, thoracic and abdominal MRA was scheduled, revealing tortuosity and ectasia of carotid, vertebral, and cerebral arteries, and marked tortuosity of two segmental pulmonary arteries (Figure 1B). No other vascular abnormalities were detected.

The proband reported that her first daughter presented tall stature $(1,76 \mathrm{~m})$ for the familial target of $1,58 \mathrm{~m}$, hypotonia at birth, congenital talipes, umbilical hernia, conservative treatment for scoliosis with orthopedic corset at 13 years, chronic articular pain, recurrent dislocations, flat feet, hallux valgus needing surgery, easy bruising, and varicose veins. On evaluation at 34 years, the second daughter presented tall stature for the familial target $(1,78 \mathrm{~m})$, high arched palate, myopia, astigmatism, mild hyperextensible skin over the neck and the elbows, atrophic post-surgical scar over the knee, arachnodactyly, mild pectus excavatum, scoliosis, joint hypermobility according to the Beighton score (6/9) (Figure 1C). She referred recurrent right patella dislocations, meniscus and lateral ligaments tear requiring arthroscopic surgery in her youth, myopia, astigmatism, easy bruising and Hashimoto thyroiditis. An ECG and echocardiography revealed normal aortic root diameter, therefore excluding ectasia or aneurysm at aortic root level. Valvular system and systolic function (EF 75\%) were normal, except for MVP with minimal regurgitation.

\section{Molecular characterization}

After written informed consent of the proband, mutational screening of the four LDS causative genes, i.e., TGFBR1, TGBFR2, SMAD3 and TGFB2, was performed. This analysis revealed the presence of the novel c.839-1G>A splice mutation in intron 5 of the TGFB2 gene, thus confirming the diagnosis of LDS4 (Figure 2A). The causal mutation was also found in the 34-year-proband's daughter. All of 

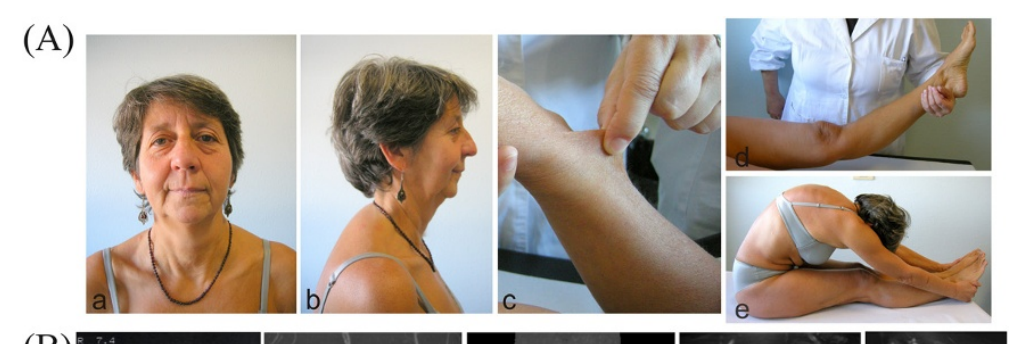

(B)

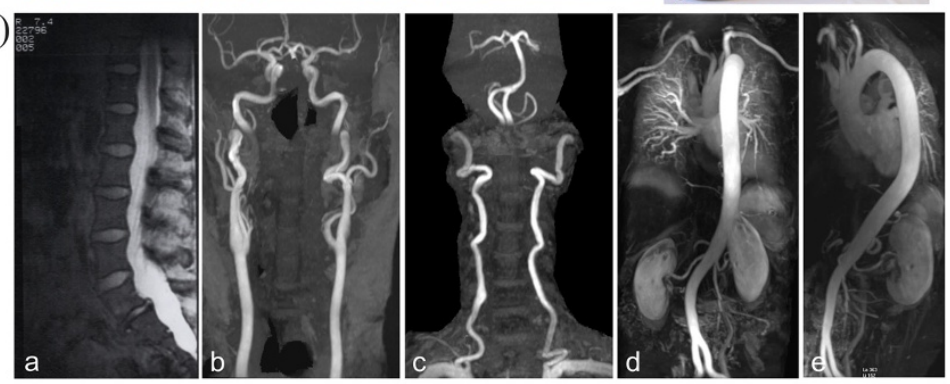

(C)
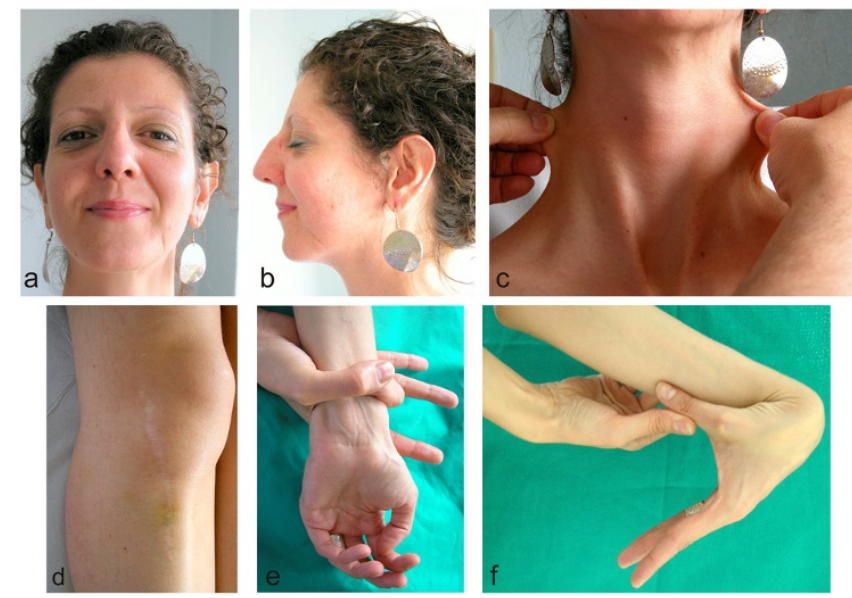

Figure 1 Clinical and radiological features of the proband and her daughter. (A) Clinical phenotype of the proband including microretrognathia and elongated philtrum $(\mathrm{a}, \mathrm{b})$, mild generalized skin hyperextensibility $(\mathrm{c})$, joint hypermobility $(\mathrm{d}, \mathrm{e})$. (B) MRI in the proposita showed dural ectasia of the lumbar tract (a), MRA disclosed tortuosity and ectasia of carotid, vertebral, and cerebral arteries (b, c) and marked tortuosity of both segmental pulmonary arteries (d). (C) Clinical presentation of the 34 years-old proband's daughter: hypotelorism, elongated philtrum and malar hypoplasia (a, b), mild skin hyperextensibility over the neck (c), atrophic post-surgical scar and ecchymosis (d), arachnodactyly with positive wrist and thumb signs (e, f).

the splicing evaluation programs of the Alamut software predicted that the c.839-1G>A mutation abolishes the canonical splice acceptor site. To verify the effect of the splicing mutation, RT-PCR analysis was performed on RNA purified from proband's blood. Amplification of the cDNA region covering exons 5-7 demonstrated that the c.839-1G>A mutation leads to the activation of a cryptic splice acceptor site $115 \mathrm{bp}$ downstream of the consensus site, generating a frameshift and a premature termination codon formation (PTC) in exon 7 (p.Gly280Aspfs*41) (Figure 2B). Furthermore, because of the lower amount of the aberrant band, compared to the wild type transcript, it is likely that the c.839-1G>A mutation activates to a certain degree the nonsense mediated RNA decay (NMD) pathway, finally resulting in TGF- $\beta 2$ haploinsufficiency (Figure 2B).

\section{Discussion}

LDS4 is a recently delineated autosomal dominant condition presenting with aneurysms, dissections, and tortuosity throughout the arterial tree in association with mitral valve disease, mild craniofacial features, and skeletal and cutaneous anomalies [16-19]. Mutations in the TGFB2 gene accounted for $25 \%$ in Lindsay et al., $1.5 \%$ in Boileau et al., $4 \%$ in Renard et al., and 1.1\% in Leutermann et al., of sampled familial cases of thoracic aortic disease that were not attributed to other known TAAD-causing genes [16-19]. So far, clinical data on LDS4 are available in a limited number of cases, i.e., 11 families and 8 sporadic patients all carrying TGFB2 mutations (Table 1 and Figure 3). Altogether, 15 independent mutations were identified, 11 of which were whole-gene deletions, frameshifts or nonsense mutations that were predicted 


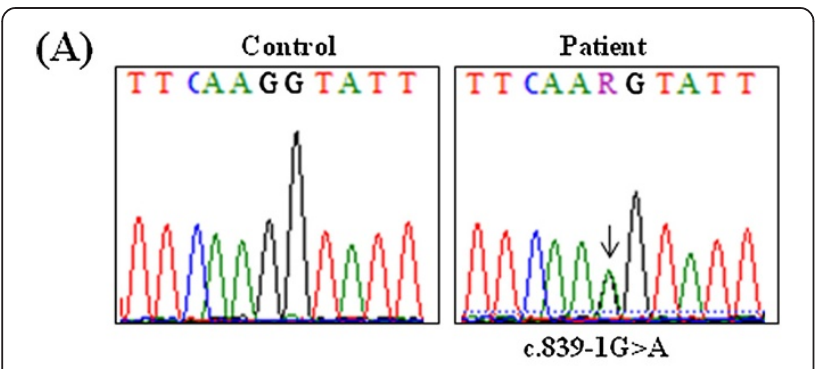

(B)

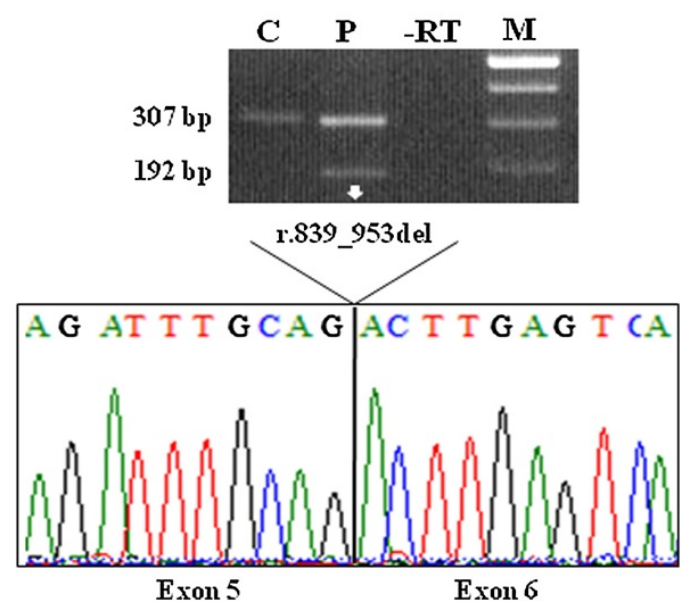

Figure 2 Molecular characterization of the proband. (A) The sequence analysis of the TGFB2 gene disclosed the novel c.839-1G>A transition (arrow), affecting the splice acceptor site of exon 6. (B) Characterization of the splicing effect of the c.839-1G>A mutation: agarose gel electrophoresis of the cDNA, amplified with primers encompassing exons $5-7$, showed in the patient $(\mathrm{P})$ the presence of an aberrant band (192 bp), in addition to the wild type fragment (307 bp), which was also detected in the control (C). The sequencing of the aberrant PCR product showed the deletion of the beginning 115 bp of exon 6 (r.839_953del), demonstrating that c.839-1G>A mutation leads to the activation of a new cryptic splice acceptor site between cDNA nucleotides 953 and 954 .

to cause to a certain degree the degradation of the cognate mRNA by NMD, and 4 were missense substitution of highly conserved amino acids with a predicted pathologic effect. In the family here described, a novel splicing mutation c.839-1G>A in intron 5 was identified in the proband and in her daughter. Amplification of the cDNA region covering exons 5-7 demonstrated that the mutation leads to the activation of a cryptic splice acceptor site within exon 6, that generates a PTC and activates to a certain level the NMD pathway. As such, the pathogenic mechanism underlying all the TGFB2 mutations is most likely loss-of-function.

Comparable to several other conditions known as TGF- $\beta$ vasculopathies and presenting with arterial aneurysms, such as MFS [21], the other LDS types [9] and ATS [5], TGFB2 loss-of-function resulted in a activation in TGF- $\beta$ signaling pathway $[22,23]$, as shown by the increase of phosphorylated SMAD2 and SMAD3 (SMAD2/3) levels in aortic lesions from $T G F B 2^{+/-}$patients and $T g f b 2^{+/-}$ mice, as well as by elevated ligand levels of either TGF- $\beta 1$ [16] or TGF- $\beta 2$ [17].

Up to date a high incidence of TAA or ectasia, mainly at the aortic root level, has been reported in LDS4 (Table 2). In particular, in Boileau et al. aortic root aneurysm was detected in $74 \%$ of the cases (14/19) [17], in Linsday et al. one main pulmonary artery aneurysm was reported; but a Z-score $>2$ was detected in $93 \%$ of the cases $(14 / 15)$ [16]. In Renard et al. TAAs were present in $67 \%$ of the cases (4/6), and in Leutermann et al. aneurysms were present in two of the family members $[18,19]$. Despite this high incidence of TAA or ectasia, a lower rate and later onset of aortic dissection is noted in LDS4 compared to other LDS types [9]. Considering the LDS4 patients reported so far, an overall dissection rate of $12 \%(5 / 43)$, at a median age of 46 years, emerged. Specifically, in Boileau et al. the aortic dissection rate was $11 \%$ (2/19 at 50 and 33 years), in Linsday et al. it was $7 \%$ (1/15 at 42 years), and in Renard et al. $33 \%$ (2/6, at 60 and about 46 years) [16-18]. None of the clinically evaluated members of the family described by Leutermann et al. had aortic dissections, but the proband (at 51 years) and his brother (at 47 years) underwent aortic aneurysm surgery and the index patient's father died at 52 years after acute aortic dissection [19]. Valve sparing aortic root replacement (VSARR) for aortic root diameter between 45 and $56 \mathrm{~mm}$ was performed also in $20 \%$ of cases $(3 / 15)$ in Linsday et al., at a median age of about 41 years [16]. Cerebral arteries involvement was delineated only in few cases; in particular, in Boileau et al. an anamnestic cerebrovascular disease was reported in $30 \%$ of the cases (3/10). Specifically, one episode of anterior communicating artery aneurysm rupture and two internal carotid aneurysm dissections were described; in the other $7 \mathrm{pa}$ tients, investigated for neck and brain arteries, no aneurysm was detected [17]. In Renard et al. a severe cerebrovascular disease was documented in one patient, a 53-year-old woman who suffered from recurrent TIA, since she was 18-year-old, recurrent strokes and presence of tortuous vertebral arteries [18]. Lindsay et al. and Leutermann et al. did not report cerebrovascular events $[16,19]$.

The incidence of arterial tortuosity is not well documented, given the limited number of patient analyzed. In particular, Boileau et al. found tortuosity in 3 out of 5 of the cases analyzed (14 patients were not investigated), and Linsday et al. in 1 out of 4 of the analyzed cases (11 patients not investigated) $[16,17]$. Renard et al. identified tortuosity of the vertebral arteries in the patient with recurrent strokes, but it is unclear if the remaining patients were studied [18]. Leutermann et al. reported arterial tortuosity in both analyzed family members with the proband showing profound cerebral and cervical arterial tortuosity [19]. 
Table 1 Overview of currently known TGFB2 mutations

\begin{tabular}{|c|c|c|c|c|}
\hline Exon & ${ }^{\mathrm{a}} \mathrm{c}$-notation & ${ }^{b} p$-notation & Domain & Reference \\
\hline 1 & c.294_308del & p.Ala100_Tyr104del & LAP & {$[16]$} \\
\hline 1 & c. $297 C>$ A & p.Tyr99* & LAP & [16] \\
\hline 1 & c.304G > T & p.Glu102* & LAP & {$[17]$} \\
\hline 3 & C. $475 \mathrm{C}>\mathrm{T}$ & p.Arg159* & LAP & [18] \\
\hline 5 & C. $771 \mathrm{C}>\mathrm{A}$ & p.Cys $257^{*}$ & LAP & {$[17]$} \\
\hline 6 & c. $839-1 \mathrm{G}>\mathrm{A}$ & p.Gly280Aspfs*41 & LAP & Current study \\
\hline 6 & c.957_972dup & p.Asn325* & LAP & {$[17]$} \\
\hline 6 & c. $979 \mathrm{C}>\mathrm{T}$ & p.Arg327Trp & LAP & {$[16,18]$} \\
\hline 6 & C. $980 \mathrm{G}>\mathrm{A}$ & p.Arg327Gln & LAP & {$[18]$} \\
\hline 6 & C. $988 \mathrm{C}>\mathrm{T}$ & p.Arg330Cys & LAP & {$[16]$} \\
\hline 7 & C. 1097 C > A & p.Pro366His & Cyt & [16] \\
\hline 7 & c.1106_1110del & p.Tyr369Cysfs*26 & Cyt & {$[16,17]$} \\
\hline 7 & c.1125del & p.Gly376Glufs*17 & Cyt & {$[18]$} \\
\hline 7 & c.1165dupA & p.Ser389Lysfs*8 & Cyt & [19] \\
\hline \multicolumn{4}{|c|}{ Entire gene Chr1.hg19: g.(215,588,712)_(222,145,072)del } & {$[16]$} \\
\hline \multicolumn{4}{|c|}{ Entire gene Chr1.hg19: g.(216,672,181)_(220,202,575)del } & {$[16]$} \\
\hline
\end{tabular}

${ }^{a}$ DNA mutation numbering is based on the cDNA sequence. For cDNA numbering, +1 corresponds to the A of the ATG translation initiation codon in the reference sequence. The reference sequence is based on the GenBank Accession number: TGFB2 NM_001135599.2. ${ }^{b}$ For protein numbering, +1 corresponds to the first translated amino acid. The reference sequence is based on the GenBank Accession number: TGFB2 NP_001129071.1. Abbreviations: LAP Latency-associated peptide domain, Cyt TGF- $\beta 2$ cytokine domain.

In our 57-year old proband and in her 34-year-old daughter aortic dissection or cardiovascular surgery did not occur and aortic aneurysms were not detected so far. Although, we are conscious that the cardiovascular phenotype of her daughter is incomplete, since no MRA was performed, echocardiography excluded at least aortic root involvement, the most common site of aneurysm in LDS4 [16-19]. Furthermore, in the proband only a brain, thoracic and abdominal MRA showed tortuosity and ectasia of the carotid, vertebral, and cerebral arteries and tortuosity of two segmental pulmonary arteries. Thus, the vascular features of our family strengthen the hypothesis of a milder vascular involvement in LDS4 compared to the other LDS types. Since a late-onset aneurysmatic vasculopathy remains possible, a periodical follow-up with vascular imaging was scheduled for the proband, and MRA study was highly recommended for her daughter.

Concerning cardiovascular surgery, Boileau et al. described 4 patients in the sixth decade, between them 3 were admitted for thoracic aortic dissection at 47 years, for TAA at 59 years and for MVP at 43 years (valve prosthesis) [17]. In Linsday et al., 2 patients were 50- and 61-year-old, and the first underwent VSARR [16]. Finally, Renard et al. described the oldest patient (69 years) who experienced a type A aortic dissection surgically treated at 60 years [18]. Three patients in sixth/seventh decade are also reported in this article, one of them had mitral valve prosthesis at 56 years

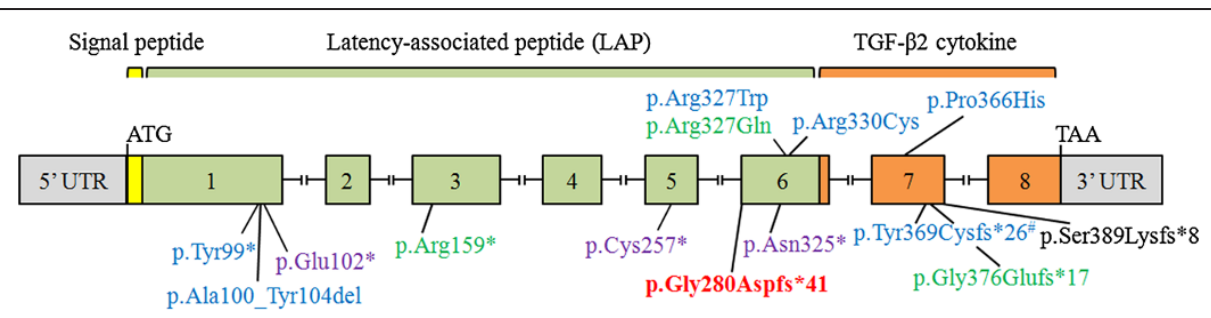

Figure 3 Overview of currently known TGFB2 mutations. Exons and untranslated regions (UTR) are represented by numbered boxes. The signal peptide is represented by a yellow, the Latency-Associated Peptide (LAP) domain by a green, and the TGF- $\beta 2$ cytokine domain by an orange box. Frameshift/truncating mutations are listed at the bottom, while missense mutations are listed on the top. The mutations reported by Boileau et al. [17] are in violet, those described by Lindsay et al. [16] in blue (" also reported by Boileau et al. [17]), those in Renard et al. [18] in green, the mutation reported in Leutermann et al. [19] is in black, and the mutation identified in this study is in red. Numbering is based on transcript NM_001135599.2 and protein reference sequence NP_001129071.1. 
Table 2 Comparison of clinical findings between the current study and those of Boileau et al. [17], Lindsay et al. [16], Renard et al. [18], and Leutermann et al. [19]

\begin{tabular}{|c|c|c|c|c|c|c|c|}
\hline \multirow[b]{2}{*}{ Clinical features } & \multirow[b]{2}{*}{ Proband } & \multirow[b]{2}{*}{ Daughter } & \multicolumn{5}{|l|}{ Frequency $^{a}$} \\
\hline & & & Boileau et al. [17] & Lindsay et al. [16] & Renard et al. [18] & Leutermann et al. [19] & Total/percentage \\
\hline \multicolumn{8}{|l|}{ Cardiovascular } \\
\hline TAA or MPA & - & $-c$ & $14 / 19$ & $1 / 4(11)$ & $4 / 6$ & $2 / 2(1)$ & $21 / 32$ (13) $65 \%$ \\
\hline Aortic dissection & - & - & $2 / 19$ & $1 / 15$ & $2 / 6$ & $0 / 3$ & $5 / 4511 \%$ \\
\hline Aortic repair & - & - & $4 / 19$ & $3 / 15$ & $0 / 6$ & $2 / 3$ & $9 / 4520 \%$ \\
\hline Arterial tortuosity & + & N.A. & $3 / 5(14)$ & $1 / 4(11)$ & $1 / 1\left(5^{d}\right)$ & $2 / 2(1)$ & $8 / 13$ (32) $61.5 \%$ \\
\hline MV impaired & + & + & $3 / 19$ & $7 / 10(5)$ & $4 / 6$ & $0 / 2(1)$ & $16 / 39$ (6) $41 \%$ \\
\hline \multicolumn{8}{|l|}{ Skeletal } \\
\hline Tall stature ${ }^{b}$ & - & + & $8 / 19$ & $11 / 15$ & N.A. & $2 / 2(1)$ & $22 / 38$ (7) $58 \%$ \\
\hline Pectus deformities & - & + & $7 / 16(3)$ & $9 / 15$ & $2 / 6$ & $1 / 3$ & $20 / 42$ (3) $47.6 \%$ \\
\hline Arachnodactyly & - & + & $8 / 13(6)$ & $8 / 15$ & $2 / 6$ & $0 / 2(1)$ & $19 / 38$ (7) 50\% \\
\hline Joint hypermobility & $+(\mathrm{BS} 9 / 9)$ & $+(\mathrm{BS} 6 / 9)$ & 10/15 (4) & $7 / 15(\mathrm{BS}>5 / 9)$ & $4 / 6$ & $2 / 3$ & $23 / 41$ (4) $56 \%$ \\
\hline Chronic Pain & + & + & N.A. & N.A. & N.A. & N.A. & $2 / 2(43)$ \\
\hline Scoliosis & + & + & 4/15 (4) & $5 / 15$ & $1 / 6$ & $3 / 3$ & $15 / 41$ (4) $36.5 \%$ \\
\hline Pes planus & - & - & $11 / 15$ (4) & $8 / 15$ & $1 / 6$ & $2 / 2(1)$ & $22 / 40$ (5) $55 \%$ \\
\hline Club feet & - & - & $0 / 15(4)$ & $5 / 15$ & $1 / 6$ & $0 / 2(1)$ & $6 / 40(5) 15 \%$ \\
\hline High arched palate & + & + & $9 / 15(4)$ & $10 / 15$ & $2 / 6$ & $1 / 2(1)$ & $24 / 40$ (5) $60 \%$ \\
\hline \multicolumn{8}{|l|}{ Cutaneous } \\
\hline Hyperelastic skin & + & + & $0 / 13(6)$ & N.A. & N.A. & N.A. & $2 / 15$ (30) $13 \%$ \\
\hline Defective scaring & - & + & N.A. & $2 / 15$ & $1 / 6$ & $0 / 2(1)$ & $4 / 25$ (20) $16 \%$ \\
\hline Easy bruising & + & + & N.A. & $5 / 11(4)$ & N.A. & $0 / 2(1)$ & $7 / 15$ (30) $46.6 \%$ \\
\hline Striae distensae & + & - & $8 / 15(4)$ & $3 / 15$ & $1 / 6$ & $0 / 2(1)$ & $13 / 40$ (5) $32.5 \%$ \\
\hline Hernia & + & - & $6 / 17(2)$ & $10 / 15$ & N.A. & $2 / 2(1)$ & $19 / 36$ (9) $52.7 \%$ \\
\hline \multicolumn{8}{|l|}{ Other } \\
\hline Dural ectasia & + & N.A. & $3 / 5(14)$ & $1 / 6(9)$ & N.A. & $0 / 2(1)$ & $5 / 14$ (31) $35.7 \%$ \\
\hline
\end{tabular}

${ }^{\mathrm{a}}$ Feature present/number of evaluated patients; in parenthesis number of patients with no data available; ${ }^{\mathrm{b}}\left(>95\right.$ th centile); ${ }^{\mathrm{C}}$ at aortic root level, ectasia and/or aneurysm of ascending aorta, aortic arch and pulmonary artery were not ascertained; ${ }^{d}$ it is unclear whether these 5 patients were investigated. BS: Beighton score; N.A. not analyzed; MV: mitral valve; TAA: thoracic aortic aneurysm; MPA: main pulmonary artery aneurysm.

for MVP [18]. Therefore, considering the age of our proband and the mean number of cardiovascular surgeries or major cardiovascular events in the patients over or in their sixth decade reported in literature, the vascular involvement in LDS4 can be even milder than described until now.

Since the tortuosity of the cerebral and neck arteries disclosed in our proband is a predisposing condition for aneurysm formation, we suggest that in LDS4 patients the vascular screening should not be restricted only to the thoracic arteries. An accurate evaluation of the brain and neck arteries, also if they might be involved only in a small percentage of patient, should be important in order to prevent severe cerebrovascular events.

Our proband and her 34-year-old daughter showed MVP, though with a low hemodynamic impairment, in agreement with the hypothesis of Renard et al. that pointed out a high rate of mitral valve disease, suggesting that this might be a signature feature of the disorder [18]. On the contrary, none of the family members reported by Leutermann et al. showed mitral valve impairment, suggesting that this hypothesis should be confirmed in larger series [19].

Concerning the musculoskeletal involvement (Table 2), Boileau et al. underlined a high rate of MFS signs, such as pes planus (73\% of cases, 11/15), high arched palate (60\% of the cases, 9/15), arachnodactyly (62\% of cases, 8/13), and pectus deformities (44\% cases, 7/16) [17]. Linsday et al., and Renard et al. confirmed the high prevalence of these signs: pes planus rate was $53 \%(8 / 15)$ and $17 \%(1 / 6)$ respectively; high arched palate rate was $67 \%(10 / 15)$ and $33 \%(2 / 6)$; arachnodactyly rate was $53 \%$ $(8 / 15)$ and $33 \%(2 / 6)$. Finally, pectus deformities rate was $60 \%(9 / 15)$ in Lindsay et al., and 33\% (2/6) in Renard et al. $[16,18]$. The musculoskeletal signs observed in the family of Leutermann et al. were scoliosis (3/3), pes 
planus (2/2), high arched palate (1/2) and joint hypermobility $(2 / 3)$ [19]. Joint hypermobility was also present in $67 \%(10 / 15)$ of the cases in Boileau et al., in $47 \%(7 / 15)$ of the cases in Linsday et al., and in 50\% (3/6) of cases in Renard et al. [16-18]. Skeletal signs of MFS were present in all members of our family. A marphanoid habitus with tall stature for the familial target, pectus excavatum, high arched palate, and arachnodactyly were recognizable in the 34-year-old daughter of the proband. In the 39-yearold daughter tall stature for the familial target, pes planus and scoliosis treated with orthopedic corset were referred. The proband only showed scoliosis and high arched palate. Both patients of our family had a positive Beighton score. Articular pain associated with joint hypermobility was not reported in literature so far in patients with LDS4, whereas both our patients referred chronic generalized articular pain. In fact, the proposita and her daughter came to our attention not for cardiovascular complains, but mainly for joint hypermobility complications: chronic articular pain since young age in the proposita, and recurrent dislocations, either in the proposita and in her daughter.

In our study, the neurological involvement, i.e., dural ectasia, was monitored only in the proposita. While the significance of dural ectasia is well recognized in MFS $[24,25]$, its prevalence in LDS is less well documented $[25,26]$. Kono et al. showed that the occurrence of dural ectasia in LDS1 and 2 was significantly higher than in controls with a frequency that varied from 40 to $70 \%$ [27]. The authors suggested that dural ectasia has the potential to become a diagnostic criterion for LDS. In support of this hypothesis, a recent comprehensive study demonstrated that dural ectasia occurs in LDS with a similar frequency and severity as in MFS [28]. Concerning LDS4 patients, Boileau et al. found dural ectasia in 3 out of 5 patients, Linsday et al. in 1 out of 10, whereas Leutermann et al., did not find this sign, and Renard et al. did not investigate it [16-19]. Given the fact that the incidence data of dural ectasia are limited to a small number of LDS4 patients (5/14, with 31 patients not analyzed), its presence should be studied in larger series of patients.

\section{Conclusions}

In conclusion, we suggest that LDS4 should be considered in patients of all ages with variable but mostly mild LDSlike phenotypes and/or with sparse signs of MFS and related connective tissue disorders with negative TGFBR1/2 and SMAD3 molecular tests, even though TAA and major cardiovascular events are absent, as it was for our proband. Molecular diagnosis allows the early identification of patients and relatives at risk of major cardiovascular complications. Further studies are needed to better delineate the clinical phenotype of LDS4 and search for new therapeutic options.

\section{Consent}

Written informed consent was obtained from patients for publication of this article and accompanying images. A copy of the written consent is available for review by the Editor-in-Chief of this journal.

\section{Abbreviations \\ CTD: Connective tissue disorders; ECG: Electrocardiogram; EF: Ejection fraction; LDS: Loeys-Dietz syndrome; MFS: Marfan syndrome; MRA: Magnetic resonance angiography; MRI: Magnetic resonance imaging; MVP: Mitral valve prolapse; NMD: Nonsense-mediated RNA decay; NSAIDs: Nonsteroidal anti-inflammatory drugs; PTC: Premature termination codon of translation; RT-PCR: Reverse-transcriptase polymerase chain reaction; SNP: Single nucleotide polymorphism; TAAD: Thoracic aortic aneurysm and dissection; VSARR: Valve sparing aortic root replacement.}

\section{Competing interests}

There are no competing interests.

\section{Authors' contributions}

MR, NC, SQ performed the molecular analyses. CD, MV, PCP, and MC made the clinical evaluation of the patients and performed the genetic counseling and follow-up. RM performed the MRA study. MR, NC and CD researched the literature, reviewed and prepared the manuscript. MR, NC and MC edited and coordinated the manuscript. All of the authors discussed, read, and approved the manuscript.

\section{Acknowledgments}

The authors thank the patients for their kind availability for this study. This study was funded by Ministero dell'Istruzione dell'Università e della Ricerca, Fondo per gli Investimenti della Ricerca di Base 2012-2013 (MIUR2012, ex fondo $60 \%$ )

\section{Author details}

${ }^{1}$ Division of Biology and Genetics, Department of Molecular and Translational Medicine, Medical Faculty, University of Brescia, Viale Europa 11, Brescia 25123, Italy. ${ }^{2}$ Department of Dermatology, University Hospital Spedali Civili, Brescia, Italy. ${ }^{3}$ Department of Radiology, University of Brescia, Brescia, Italy.

Received: 5 March 2014 Accepted: 18 July 2014

Published: 28 August 2014

\section{References}

1. Dietz HC, Cutting GR, Pyeritz RE, Maslen CL, Sakai LY, Corson GM, Puffenberger EG, Hamosh A, Nanthakumar EJ, Curristin SM, Stetten G, Meyers DA, Francomano CA: Marfan syndrome caused by a recurrent de novo missense mutation in the fibrillin gene. Nature 1991, 352:337-339.

2. Loeys BL, Chen J, Neptune ER, Judge DP, Podowski M, Holm T, Meyers J, Leitch CC, Katsanis N, Sharifi N, Xu FL, Myers LA, Spevak PJ, Cameron DE, De Backer J, Hellemans J, Chen Y, Davis EC, Webb CL, Kress W, Coucke P, Rifkin DB, De Paepe AM, Dietz HC: A syndrome of altered cardiovascular, craniofacial, neurocognitive and skeletal development caused by mutations in TGFBR1 or TGFBR2. Nat Genet 2005, 37:275-281.

3. Loeys BL, Schwarze U, Holm T, Callewaert BL, Thomas GH, Pannu H, De Backer JF, Oswald GL, Symoens S, Manouvrier S, Roberts AE, Faravelli F, Greco MA, Pyeritz RE, Milewicz DM, Coucke PJ, Cameron DE, Braverman AC, Byers PH, De Paepe AM, Dietz HC: Aneurysm syndromes caused by mutations in the TGFbeta receptor. N Engl J Med 2006, 355:788-798.

4. Van de Laar IM, Oldenburg RA, Pals G, Roos-Hesselink JW, de Graaf BM, Verhagen JM, Hoedemaekers YM, Willemsen R, Severijnen LA, Venselaar $H_{\text {, }}$ Vriend G, Pattynama PM, Collee M, Majoor-Krakauer D, Poldermans D, Frohn-Mulder IM, Micha D, Timmermans J, Hilhorst-Hofstee Y, Bierma-Zeinstra SM, Willems PJ, Kros JM, Oei EH, Oostra BA, Wessels MW, Bertoli-Avella AM: Mutations in SMAD3 cause a syndromic form of aortic aneurysms and dissections with early-onset osteoarthritis. Nat Genet 2011, 43:121-126.

5. Coucke PJ, Willaert A, Wessels MW, Callewaert B, Zoppi N, De Backer J, Fox JE, Mancini GM, Kambouris M, Gardella R, Facchetti F, Willems PJ, Forsyth R, Dietz HC, Barlati S, Colombi M, Loeys B, De Paepe A: Mutations in the facilitative glucose transporter GLUT10 alter angiogenesis and cause arterial tortuosity syndrome. Nat Genet 2006, 38:452-457. 
6. Guo DC, Pannu H, Tran-Fadulu V, Papke CL, Yu RK, Avidan N, Bourgeois S, Estrera AL, Safi HJ, Sparks E, Amor D, Ades L, McConnell V, Willoughby CE, Abuelo D, Willing M, Lewis RA, Kim DH, Scherer S, Tung PP, Ahn C, Buja LM, Raman CS, Shete SS, Milewicz DM: Mutations in smooth muscle alpha-actin (ACTA2) lead to thoracic aortic aneurysms and dissections. Nat Genet 2007, 39:1488-1493.

7. Zhu L, Vranckx R, Khau Van Kien P, Lalande A, Boisset N, Mathieu F, Wegman M, Glancy L, Gasc JM, Brunotte F, Bruneval P, Wolf JE, Michel JB, Jeunemaitre $X$ : Mutations in myosin heavy chain 11 cause a syndrome associating thoracic aortic aneurysm/aortic dissection and patent ductus arteriosus. Nat Genet 2006, 38:343-349.

8. Wang L, Guo DC, Cao J, Gong L, Kamm KE, Regalado E, Li L, Shete S, He WQ, Zhu MS, Offermanns S, Gilchrist D, Elefteriades J, Stull JT, Milewicz DM: Mutations in myosin light chain kinase cause familial aortic dissections. Am J Hum Genet 2011, 88:516.

9. Loeys BL, Dietz HC: Loeys-Dietz Syndrome. In GeneReviews ${ }^{\mathrm{TM}}$ [Internet]. Edited by Pagon RA, Adam MP, Bird TD, et al. Seattle (WA): University of Washington, Seattle; 2008:1993-2013 [Updated 2013 Jul 11].

10. Van Hemelrijk C, Renard M, Loeys B: The Loeys-Dietz syndrome: an update for the clinician. Curr Opin Cardiol 2010, 25:546-551.

11. Drera B, Ritelli M, Zoppi N, Wischmeijer A, Gnoli M, Fattori R, Calzavara-Pinton PG, Barlati S, Colombi M: Loeys-Dietz syndrome type I and type II: clinical findings and novel mutations in two Italian patients. Orphanet J Rare Dis 2009, 2:4. 24.

12. Van Laer L, Dietz H, Loeys B: Loeys-Dietz syndrome. Adv Exp Med Biol 2014, 802:95-105

13. Regalado ES, Guo DC, Villamizar C, Avidan N, Gilchrist D, McGillivray B, Clarke L, Bernier F, Santos-Cortez RL, Leal SM, Bertoli-Avella AM, Shendure J, Rieder MJ, Nickerson DA, NHLBI GO, Exome Sequencing Project, Milewicz DM: Exome sequencing identifies SMAD3 mutations as a cause of familial aortic aneurysm and dissection with intracranial and other arterial aneurysms. Circ Res 2011, 109:680-686.

14. Van de Laar IM, Van der Linde D, Oei EH, Bos PK, Bessems JH, Bierma-Zeinstra SM, van Meer BL, Pals G, Oldenburg RA, Bekkers JA, Moelker A, de Graaf BM, Matyas G, Frohn-Mulder IM, Timmermans J, Hilhorst-Hofstee Y, Cobben JM, Bruggenwirth HT, van Laer L, Loeys B, De Backer J, Coucke PJ, Dietz HC, Willems PJ, Oostra BA, De Paepe A, Roos-Hesselink JW, Bertoli-Avella AM, Wessels MW: Phenotypic spectrum of the SMAD3-related aneurysms-osteoarthritis syndrome. J Med Genet 2012, 49:47-57.

15. Wischmeijer A, Van Laer L, Tortora G, Bolar NA, Van Camp G, Fransen E, Peeters N, di Bartolomeo R, Pacini D, Gargiulo G, Turci S, Bonvicini M, Mariucci E, Lovato L, Brusori S, Ritelli M, Colombi M, Garavelli L, Seri M, Loeys BL: Thoracic aortic aneurysm in infancy in aneurysms-osteoarthritis syndrome due to a novel SMAD3 mutation: further delineation of the phenotype. Am J Med Genet A 2013, 161A:1028-1035.

16. Lindsay ME, Schepers D, Bolar NA, Doyle JJ, Gallo E, Fert-Bober J, Kempers MJ, Fishman EK, Chen Y, Myers L, Bjeda D, Oswald G, Elias AF, Levy HP Anderlid BM, Yang MH, Bongers EM, Timmermans J, Braverman AC, Canham N, Mortier GR, Brunner HG, Byers PH, Van Eyk J, Van Laer L, Dietz HC, Loeys BL: Loss-of-function mutations in TGFB2 cause a syndromic presentation of thoracic aortic aneurysm. Nat Genet 2012, 44:922-927.

17. Boileau C, Guo DC, Hanna N, Regalado ES, Detaint D, Gong L, Varret M, Prakash SK, Li AH, D'Indy H, Braverman AC, Grandchamp B, Kwartler CS, Gouya L, Santos-Cortez RL, Abifadel M, Leal SM, Muti C, Shendure J, Gross MS, Rieder MJ, Vahanian A, Nickerson DA, Michel JB, National Heart, Lung, and Blood Institute (NHLBI) Go Exome Sequencing Project, Jondeau G, Milewicz DM: TGFB2 mutations cause familial thoracic aortic aneurysms and dissections associated with mild systemic features of Marfan syndrome. Nat Genet 2012, 44:916-921.

18. Renard M, Callewaert B, Malfait F, Campens L, Sharif S, Del Campo M, Valenzuela I, Mcwilliam C, Coucke P, De Paepe A, De Backer J: Thoracic aortic-aneurism and dissection in association with significant mitral valve prolapse cause by mutation in TGFB2. Int J Cardiol 2013, 165:584-587.

19. Leutermann R, Sheikhzadeh S, Brockstädt L, Rybczynski M, van Rahden V, Kutsche K, von Kodolitsch Y, Rosenberger G: A 1-bp duplication in TGFB2 in three family members with a syndromic form of thoracic aortic aneurysm. Eur J Hum Genet 2013, 22(7):944-948.

20. MacCarrick G, Black JH 3rd, Bowdin S, El-Hamamsy I, Frischmeyer-Guerrerio PA, Guerrerio AL, Sponseller PD, Loeys B, Dietz HC 3rd: Loeys-Dietz syndrome: a primer for diagnosis and management. Genet Med 2014, doi:10.1038/gim.2014.11. [Epub ahead of print].
21. Neptune ER, Frischmeyer PA, Arking DE, Myers L, Bunton TE, Gayraud B, Ramirez F, Sakai LY, Dietz HC: Dysregulation of TGFbeta activation contributes to pathogenesis in Marfan syndrome. Nat Genet 2003, 33:407-411.

22. Moustakas $\mathrm{A}$, Heldin $\mathrm{CH}$ : The regulation of TGFbeta signal transduction. Development 2009, 136:3699-3714.

23. Akhurst RJ: The paradoxical TGF- $\beta$ vasculopathies. Nat Genet 2012, 44:838-839.

24. De Paepe A, Devereux RB, Dietz HC, Hennekam RC, Pyeritz RE: Revised diagnostic criteria for the Marfan syndrome. Am J Med Genet 1996 62:417-426.

25. Loeys BL, Dietz HC, Braverman AC, Callewaert BL, De Backer J, Devereux RB, Hilhorst-Hofstee $Y$, Jondeau G, Faivre L, Milewicz DM, Pyeritz RE, Sponseller PD, Wordsworth P, De Paepe AM: The revised Ghent nosology for the Marfan syndrome. J Med Genet 2010, 47:476-485.

26. Rodrigues VJ, Elsayed S, Loeys BL, Dietz HC, Yousem DM: Neuroradiologic manifestations of Loeys-Dietz syndrome type 1. Am J Neuroradiol 2009, 30:1614-1619.

27. Kono AK, Higashi M, Morisaki H, Morisaki T, Naito H, Sugimura K: Prevalence of dural ectasia in loeys-dietz syndrome: comparison with marfan syndrome and normal controls. PLoS One 2013, 8:e75264.

28. Sheikhzadeh S, Brockstaedt L, Habermann CR, Sondermann C, Bannas P, Mir TS, Staebler A, Seidel H, Keyser B, Arslan-Kirchner M, Kutsche K, Berger J, Blankenberg S, von Kodolitsch Y: Dural ectasia in Loeys-Dietz syndrome: comprehensive study of 30 patients with a TGFBR1 or TGFBR2 mutation. Clin Genet 2013, doi:10.1111/cge.12308.

doi:10.1186/s12881-014-0091-8

Cite this article as: Ritelli et al:: Further delineation of Loeys-Dietz syndrome type 4 in a family with mild vascular involvement and a TGFB2 splicing mutation. BMC Medical Genetics 2014 15:91.

\section{Submit your next manuscript to BioMed Central and take full advantage of:}

- Convenient online submission

- Thorough peer review

- No space constraints or color figure charges

- Immediate publication on acceptance

- Inclusion in PubMed, CAS, Scopus and Google Scholar

- Research which is freely available for redistribution

Submit your manuscript at www.biomedcentral.com/submit
C Biomed Central 